\title{
Sarcoidosis and multiple sclerosis: systemic toxicity associated with the use of interferon-beta therapy
}

\author{
C. Carbonelli1, S. Montepietra², A. Caruso3, A. Cavazza4, \\ C. Feo², F. Menzella1, L. Motti2, L. Zucchi1
}

ABSTRACT: Sarcoidosis and multiple sclerosis: systemic toxicity associated with the use of interferon-beta therapy. C. Carbonelli, S. Montepietra, A. Caruso, A. Cavazza, C. Feo, F. Menzella, L. Motti, L. Zucchi.

Sarcoidosis is a multi-systemic inflammatory disease of unknown origin characterized by the presence of noncaseating epitheloid cell granulomas in multiple organs. Diagnosis is made on the basis of a compatible clinical-radiological scenario and the histological demonstration of the typical granulomas in the affected tissues. Interferons are immuno-modulators that have been used in a wide range of diseases, including hepatitis $\mathrm{C}$ virus infection, multiple sclerosis, and multiple myeloma and other types of tumours, including leukemia, lymphomas, Kaposi's sarcoma, and melanoma. Interferon- $\alpha$-induced sarcoidosis has been reported repeatedly and there are two re- ports in the literature of cases of pulmonary sarcoidosis treated with interferon-1b therapy: one for advanced renal cell carcinoma and the other for multiple myeloma. A 35-year-old man on chronic immune-modulant Interferon-1b-based therapy for multiple sclerosis presented to the Neurology Unit with mild dyspnoea, dry cough, and transient pain to right upper abdomen. Lungs, spleen, liver, and almost all lymphnode stations of abdomen and mediastinum were clearly involved on ultrasound examination, chest X-ray, and computed tomography. A transbronchial biopsy showed non-caseating granuloma on histopathologic evaluation of the lungs. To the best of our knowledge, this is the first report of a chronic multisystemic sarcoidosis that was associated with interferon-beta treatment.

Monaldi Arch Chest Dis 2012; 77: 1, 29-31.

Keywords: Multiple sclerosis, Demyelinating diseases, Neurological disorders, Adverse effects, Medication, Management, Interferons, Sarcoidosis.

I Pulmonology Unit, Department of Cardiology, Thoracic and Vascular Surgery and Critical Care Medicine, Azienda Ospedaliera ASMN, Istituto di Ricovero e Cura a Carattere Scientifico, Reggio Emilia,

2 Neurology Unit, Department of Neuromotor Physiology, Azienda Ospedaliera ASMN, Istituto di Ricovero e Cura a Carattere Scientifico, Reggio Emilia,

3 Rheumatology Unit, Departement of Internal Medicine, Azienda Ospedaliera ASMN, Istituto di Ricovero e Cura a Carattere Scientifico, Reggio Emilia,

4 Pathology Unit, Department of Oncology, Azienda Ospedaliera ASMN, Istituto di Ricovero e Cura a Carattere Scientifico, Reggio Emilia, Italy.

Correspondence: Cristiano Carbonelli, MD; Pulmonology Unit, Department of Cardiology, Thoracic and Vascular Surgery and Critical Care Medicine, Azienda Ospedaliera ASMN, Istituto di Ricovero e Cura a Carattere Scientifico, Viale Risorgimento 80, 42123 Reggio Emilia, Italy; e-mail: cristiano.carbonelli@asmn.re.it

\section{Case report}

A 35-year-old Caucasian male with no previous illnesses was admitted to the hospital due to complaint of hyposthenia to the left part of the body and slow and progressive onset of paresthesias from the big toe to the lateral cervical and retro auricular region.

On physical examination, no cranial district involvement was found, there was a slight pronation to the Barré on the left, left prevalence of osteotendinous reflexes with extension of reflexogenic area, hypoaesthesia tactile-sensory of the left part of the body. The Magnetic Resonance (MR) image was consistent with a demyelinating process, the compatible evocated potentials findings, and $\operatorname{IgG}$ presence in the cerebrospinal fluid all supported a diagnosis of multiple sclerosis (MS).

The clinical and instrumental evaluation of chest and abdomen at onset and at subsequent visits did not reveal any abnormality suggestive of a differential diagnosis.

Continuous therapy with systemic corticosteroids did not control the disease and the patient complained of a further episode of sensitive reactivation six months later. MR and clinical McDonald criteria for MS were met (multiple clinical attacks and MR showing typical lesion dissemination in space). Interferon- $1 \beta$ (IFN-1 $\beta$ ) therapy was thus initiated (22 mcg sc tiw), resulting in clinical remission of the symptoms. This therapy was suspended 2 months later as the patient and his partner 
wanted to have a baby and were concerned about the possible effects taking this medication might have on a pregnancy. Four months later, an episode of optical neuritis was classified as further MS reactivation, and treatment with IFN-1 $\beta 22 \mathrm{mcg}$ tiw was therefore resumed. Six years later, mild dyspnoea developed gradually, with dry cough and transient pain to right upper abdomen exacerbated by deep breathing. There was no fever, weight loss, or night sweats, blood tests revealed abnormalities involving the increase of serum aminotranferase levels, hypoalbuminemia and hypergammaglobulinemia were found, and urine analysis revealed a marked hypercalciuria.

Abdominal ultrasonography showed hepatosplenomegaly with multiple hypodense lesions. A chest X-ray showed bilateral hilar and right paratracheal adenopathy and diffuse reticulonodular disease pattern in the lungs.

A CT scan evaluation and a total body $18 \mathrm{~F}$ FDG-PET confirmed the systemic spread of the disease, with the enlargement of most of the abdominal and mediastinal lymphnode stations, the involvement of the spleen (figure 1) and the liver entirely, and of the upper zone of lung parenchyma, with a micronodular pattern.

A bronchoscopy was performed to obtain bronchial and transbronchial biopsies, which demonstrated the presence of non caseating granuloma typical of sarcoidosis (figure 2).

The patient was thought to have a systemic toxicity due to IFN-1 $\beta$, which was therefore suspended.

Due to the persistence of the disease after three months of follow up, treatment with prednisone and hydroxychloroquine was initiated. Hematologic laboratory exams improved and marked improvement in ultrasonography findings was noted after 1 month. The described treatment led to the complete resolution of the granulomatous disease; sarcoidosis has not recurred over these last 24 months although neurologic, clinical, and MR assessments have consistently shown an unaltered MS scenario.

\section{Discussion}

Sarcoidosis is a disease of unknown cause whose diagnosis is made based on histological and radiological findings and when its typical clinical manifestations are present, although these may vary and may even be absent in half of cases at diagnosis [1]. Several pathophysiological mechanisms have been proposed to explain the lung damage produced by IFN, focusing on its known immuno-modulatory activity [2]. The spectrum of lung tissue damage associated with the use of IFNs is very broad. The association of sarcoidosis with INF- $\alpha$ treatment for HCV infection is well-described [3]. The possibility that IFN-1 $\beta$ causes sarcoidosis was already described long ago. To our knowledge, two cases of pulmonary sarcoidosis have been described in the course of INF- $\beta$ therapy, one following interferon therapy for advanced renal cell carcinoma and the other with interferon-

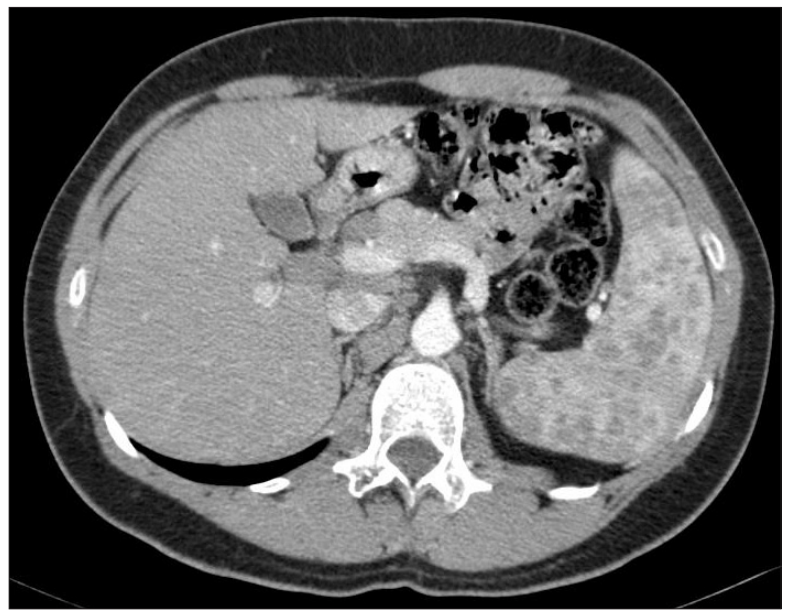

Fig. 1. - Multinodular involvement of the spleen seen at CT.

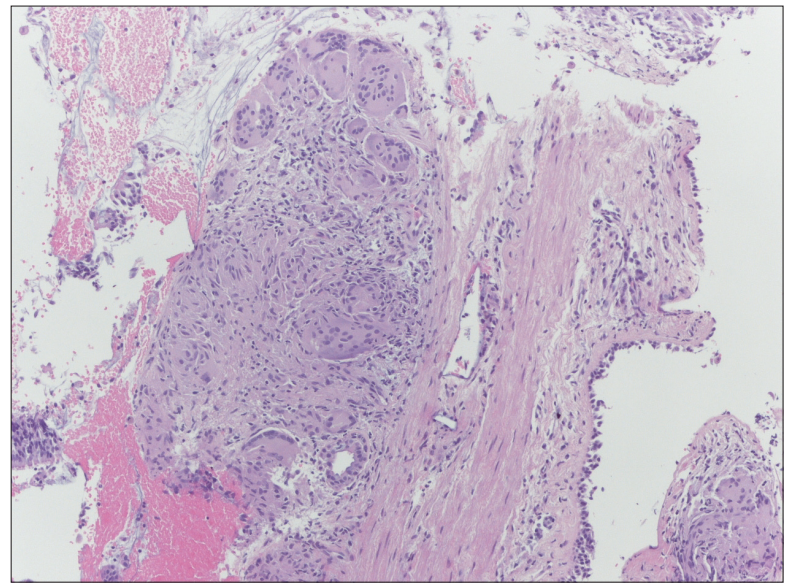

Fig. 2. - A well-formed non necrotising granuloma with giant cells is present in the bronchial mucosa (Haematoxylin-Eosin, 100X).

beta 1 therapy for multiple myeloma $[4,5]$. Symptoms of IFN-1 $\beta$-induced sarcoidosis were subtle at diagnosis for these cases of limited pulmonary sarcoidosis, as they were in our case of chronic multisystemic disease. The available literature reports the average duration of IFN therapy at the time of the diagnosis of sarcoidosis as being 34 weeks (range 2-168 weeks); the cases described are mostly related to the use of recombinant IFN$\alpha 2 \mathrm{~b}$, at a dose of 3 MIU subcutaneously (sc) tiw [6]. In our patient, instead, sarcoidosis appeared 288 weeks after the initiation of a dose of $22 \mathrm{mcg}$ of IFN-1 $\beta$ sc tiw.

Historically, the medical literature has addressed the misdiagnosis of MS by referring primarily to other inflammatory processes, with Neurosarcoidosis (NS) being the most commonly encountered disease initially diagnosed as MS. Classic isolated neurologic syndromes typically occurring as the first manifestations of MS are also common in NS, as well as cerebrospinal fluid abnormalities like oligoclonal bands and elevated IgG concentration. Further, the McDonald MR criteria for lesion dissemination in space are of little help in distinguishing between MS and NS [7]. Neurologists tend to doubt the coexistence of MS and systemic sarcoidosis, changing their diagnosis to NS whenever a biopsy proving systemic sarcoido- 
sis is available. In our case, we emphasise the possibility that another confounding element may be represented by the treatment itself, which can determine sarcoidosis as chronic multisystemic complication of a therapy for MS.

We performed three months of follow up after the diagnosis of systemic sarcoidosis and the withdrawal of IFN-1 $\beta$ therapy without a spontaneous remission of the disease. This, along with the longer latency observed in our case, goes against the clinical logic that sarcoidosis was pharmacologically induced, as pulmonary toxicities usually disappear spontaneously after the withdrawal of the responsible drug. On the other hand, the complete regression of the disease with steroids without recurrences led us to conclude that what we observed was not an idiopathic process but a disease secondary to drug toxicity. Repeated clinical and MR assessments showing an unaltered MS scenario confirmed our hypothesis of chronic multisystemic sarcoidosis as a complication of interferon treatment for MS.

In conclusion, the case here described demonstrates that chronic multi-systemic sarcoidosis can be a complication of interferon-based treatment for MS and that the collaboration between pulmonologists and neurologists is thus crucial. Moreover, it is well known that NS is often indistinguishable from MS at presentation. Ours is the first report of a multi-organ sarcoidosis that we concluded was associated with interferon-beta treatment. As symptoms of sarcoidosis are subtle at diagnosis and there is a wide variety of possible manifestations, with extra pulmonary involvement more likely when the disease is drug-induced, this clinical possibility must be taken into account.

\section{References}

1. Costabel U, Ohshimo S, Guzman J. Diagnosis of sarcoidosis. Curr Opin Pulmon Med 2008; 14: 455-461.

2. Baron S, Tyring S, Fleischmann WR Jr, et al. The interferons: mechanisms of action and clinical applications. JAMA 1991; 266: 1375-83.

3. Goldber HJ, Fielder D, Webb A, et al. Sarcoidosis after treatment with interferon-alfa: a case series and rewiew of the literature. Resp Med 2006; 100: 2063-68.

4. Bobbio-Pallavicini E, Valsecchi C, Tacconi F, et al. Sarcoidosis following beta-interferon therapy for multiple myeloma. Sarcoidosis 1995; 12: 140-142.

5. Abdi EA Nguyen GK, Ludwig RN, Dickour WJ. Pulmonary sarcoidosis following interferon therapy for advanced renal cell carcinoma. Cancer 1987; 59: 896-900.

6. Alazemi S, Campos MA. Interferon-induced sarcoidosis. Int J Clin Pract 2006 Feb; 60: 201-11.

7. Scott TF, Yandora K, Kunschner LJ, et al. Neurosarcoidosis mimicry of multiple sclerosis: clinical, laboratory, and imaging characteristics. Neurologist 2010; 16: 386-9.

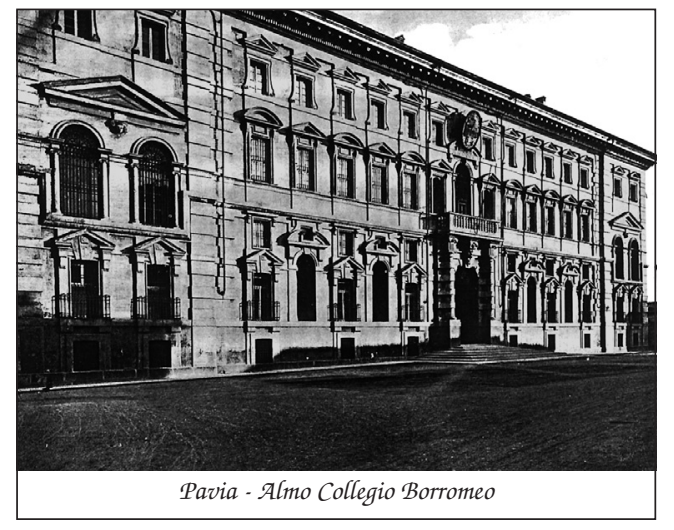

